\title{
Retrospective Analysis of Treatment and Complications of Immune Checkpoint Inhibitor-Associated Colitis: Histological Ulcerations as Potential Predictor for a Steroid-Refractory Disease Course
}

\author{
Julian Burla ${ }^{a}$ Sena Bluemel ${ }^{a} \quad$ Luc Biedermann $^{a} \quad$ Marjam J. Barysch ${ }^{b}$ \\ Reinhard Dummer $^{\mathrm{b}}$ Mitchell P. Levesque ${ }^{\mathrm{b}}$ Christoph Gubler ${ }^{\mathrm{a}}$ \\ Bernhard Morell ${ }^{\mathrm{a}}$ Gerhard Rogler ${ }^{\mathrm{a}} \quad$ Michael Scharl ${ }^{\mathrm{a}}$ \\ aDivision of Gastroenterology and Hepatology, University Hospital Zurich, University of Zurich, Zurich, Switzerland; \\ ${ }^{b}$ Department of Dermatology, University Hospital Zurich, University of Zurich, Zurich, Switzerland
}

\section{Keywords}

Malignant melanoma $\cdot$ Histology $\cdot$ Infliximab ·

Vedolizumab $\cdot$ Nivolumab

\begin{abstract}
Background/Aims: Among the severe immune-related adverse events (irAEs) that occur with immune checkpoint inhibitor (ICI) therapy, colitis is the most frequent one. This study aimed at describing the experience from the largest gastroenterology unit in Switzerland with immune checkpoint inhibitor-associated colitis (ICIAC), its clinical presentation, management, and outcomes. Methods: We performed a retrospective review of patients who were referred for the evaluation of ICIAC between January 2011 and October 2018 to the Division of Gastroenterology and Hepatology, University Hospital Zurich. Results: Thirty-three patients with immune-related colitis grade 3 or 4 met the inclusion criteria and were analyzed in detail: All patients had diarrhea, $64 \%$ had abdominal pain, $42 \%$ had bloody stool, $27 \%$ had emesis, and $18 \%$ developed fever. In total, $33 \%$ were successfully treated with corticosteroids alone; $66 \%$ were steroid-refrac-
\end{abstract}

karger@karger.com www.karger.com/iid

Karger"
(C) 2020 The Author(s)

Published by S. Karger AG, Basel

Karger

Open access

This is an Open Access article licensed under the Creative Commons Attribution-NonCommercial-4.0 International License (CC BY-NC) (http://www.karger.com/Services/OpenAccessLicense), applicable to the online version of the article only. Usage and distribution for commercial purposes requires written permission. tory and treated with infliximab or vedolizumab. Two of these patients developed severe complications requiring surgery. All patients reached complete remission of ICIAC and its symptoms. At colonoscopy, ulcerations were seen in $37 \%$ of steroid-refractory versus $63 \%$ of steroid-responsive cases. Deep histological ulcerations invading the submucosa were only present in steroid-refractory cases. Conclusion: ICIAC is a severe irAE which frequently requires high-dose steroids and a close follow-up due to deleterious complications. The detection of histologically diagnosed deep ulcerations may predict a steroid-refractory course and may warrant early application of infliximab. However, larger studies are required to confirm our findings.

(c) 2020 The Author(s).

Published by S. Karger AG, Basel

\section{Introduction}

Checkpoint inhibitor molecules, such as ipilimumab, nivolumab, and pembrolizumab, are a successful new approach in cancer therapy. These antibodies block inhibitory receptor proteins on the surface of $\mathrm{T}$ lymphocytes, 
which are, otherwise, stimulated by cancer cells to obtain immune tolerance [1]. As a result, these drugs activate the immune system and trigger an immunogenic response against multiple malignancies with melanoma representing the best evaluated indication $[2,3]$. Available antibodies target CTLA-4 (cytotoxic T lymphocyte antigen 4), PD-1 (programmed cell death receptor-1), or PD-1 ligand 1 (PD-L1) [1-3]. Ipilimumab (anti-CTLA-4) is used for the treatment of melanoma, while pembrolizumab, nivolumab (both anti-PD-1), and atezolizumab (antiPD-L1) are additionally used in renal cell carcinoma, non-small cell lung cancer, urothelial cancer, and some other tumor types [4-9].

Nevertheless, checkpoint blockade may also lead to a loss of immune tolerance of healthy tissue [2]. This may result in a variety of side effects, referred to as immunerelated adverse events (irAEs). Besides skin rash, colitis is the most frequent irAE [10]. Overall, 15-25\% of patients on single immunotherapy and about $65 \%$ of patients on combined immunotherapy suffer from grade 3 or grade 4 irAEs [11, 12]. Up to $22 \%$ of patients on ipilimumab monotherapy and about $25 \%$ of patients on combined therapy suffer from severe (grade 3 or 4 ) enterocolitis that requires treatment interruption as well as high-dose steroids and/or anti-TNF antibodies (infliximab) or even colectomy [12]. In general, most cases are mild to moderate and respond to corticosteroids [13].

In this retrospective single-center analysis, we summarize our experiences with immune checkpoint inhibitorassociated colitis (ICIAC) and discuss clinical features, its management, and outcomes.

\section{Methods}

\section{Study Cohort}

We performed a retrospective review of patient charts from patients who were treated with ipilimumab alone or in combination with pembrolizumab or nivolumab and were referred to the Division of Gastroenterology and Hepatology, University Hospital Zurich, for the diagnostic evaluation of diarrhea between January 2011 and October 2018. We included cases who (1) developed ICIAC, (2) were diagnosed with melanoma, and (3) had received one or more cycles of ICI at the Department of Dermatology at University Hospital Zurich. ICIAC is defined as colitis due to ICI therapy excluding other causes of bowel inflammation and diarrhea. Checkpoint inhibitor doses and courses were chosen according to the standardized treatment regime approved by the Swiss health authorities. In case of drug toxicity, disease progression or death treatment was not completed.

\section{Patient Data}

All patients' histories were profoundly assessed. We collected demographic and clinical data including sex, age, tumor stage according to the American Joint Committee on Cancer (AJCC), type of therapy with checkpoint inhibitors, treatment courses, colitis and its symptoms, other associated irAEs, laboratory results, stool examinations, endoscopic findings, and histology reports of conducted biopsies. For tumor staging (with prognostic relevance), the AJCC guidelines classify melanomas according to invasion depth into the following stages: $<2.0 \mathrm{~mm}$ having no melanoma ulceration (stage I), $>1.0-2.0 \mathrm{~mm}$ with ulceration and $>2.0-4.0 \mathrm{~mm}$ without ulceration (stage IIA), $>2.0-4.0 \mathrm{~mm}$ with ulceration and $>4.0 \mathrm{~mm}$ without ulceration (stage IIB), $>4.0 \mathrm{~mm}$ with ulceration (stage IIC), any tumor depth with micrometastases/lymph node metastases or in-transit metastases (stage III), and any tumor depth with distant metastases (stage IV). Details can be found here [14]. Colitis was treated according to the current guidelines [15]. Patients were grouped into steroid-responsive patients, who received only prednisone with or without budesonide to achieve complete remission, and steroid-refractory cases. We defined steroid-refractory as colitis requiring treatment escalation with infliximab, vedolizumab, or surgery.

The severity of diarrhea and colitis was graded according to the Common Terminology Criteria for Adverse Events (CTCAE), version 4.03. Grade 1 diarrhea is defined as less than 4 bowel movements per day over baseline, grade 2 as $4-6$ bowel movements, grade 3 as 7 or more bowel movements, and grade 4 as life-threatening consequences, with urgent intervention indicated. Grade 1 colitis is asymptomatic (diagnostic observations only); grade 2 is associated with blood or mucus in the stool; grade 3 is defined as a change of bowel habits or severe abdominal pain; grade 4 indicates life-threatening consequences, for example, colonic perforations; and grade 5 represents death. Patients' endoscopy reports including image documentation were reviewed to analyze the distribution and characteristics of colitis. We defined "extensive colitis" as endoscopically detected inflammation on both sides of the colon (right colon: cecum and ascending colon; left colon: descending and sigmoid colon). Ulcerative colitis (macroscopic ulcers of any size or quantity) was separated from non-ulcerative inflammation. The histological diagnosis of an ulceration is defined as a lesion extending beyond the muscularis mucosae into the submucosa [16].

\section{Data Presentation}

Descriptive data are summarized as a number with percentage for nominal or median with range for continuous variables, respectively. Analyses were performed with the SPSS software package, version 25 (IBM Corp., Armonk, NY, USA). Due to the small sample size, a statistical comparison between patients with different colitis stages and/or a steroid refractory/responsive course does not appear to be valid and was therefore not performed.

\section{Results}

We identified 49 patients who were treated with ipilimumab alone or in combination with pembrolizumab or nivolumab and were referred to the Division of Gastroenterology and Hepatology, University Hospital Zurich, 
Fig. 1. Flow chart of study inclusion and

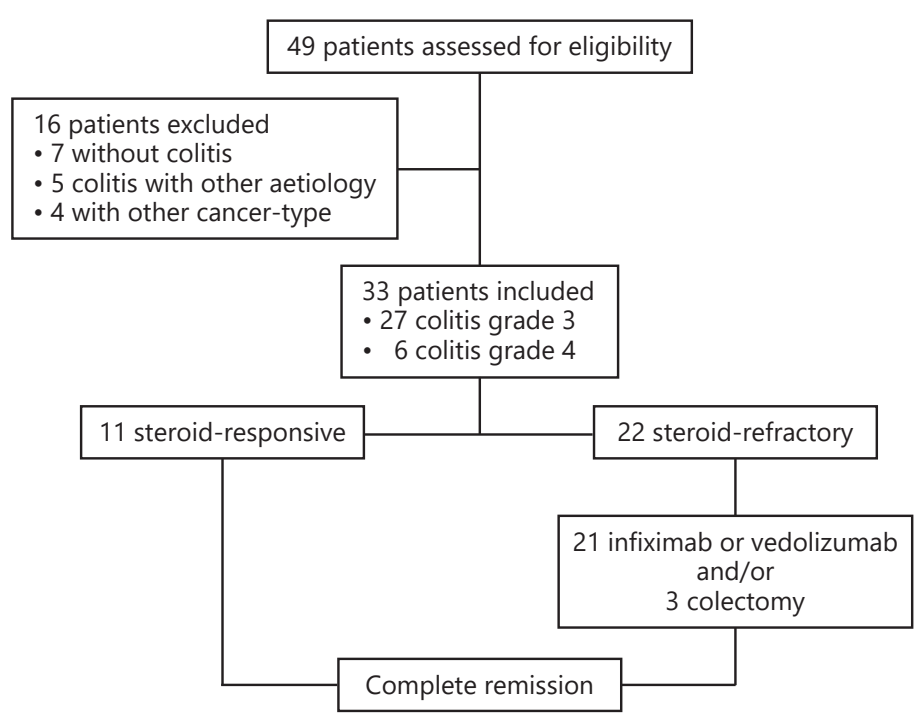
outcome.

Switzerland, for diagnostic evaluation of diarrhea. We finally included 33 patients who matched the inclusion criteria (Fig. 1). Thirty patients underwent microbiological stool testing. An infectious cause of colitis was ruled out in $97 \%$. One patient tested positive for Clostridium difficile toxin and was treated with metronidazole. After persistence of symptoms despite antibiotic treatment, the patient was diagnosed with checkpoint inhibitor-induced colitis and needed additional treatment with corticosteroids and infliximab to recover.

\section{Tumor Stage and Checkpoint Inhibitor Therapy}

Detailed information about tumor stage and treatment is given in Table 1. All included patients with melanoma AJCC stage III or IV [14] developed immune-related colitis and diarrhea. Most were treated with ipilimumab alone. About one-third additionally received pembrolizumab or nivolumab. Twenty-five patients were evaluated according to the response evaluation criteria in solid tumors (RECIST): The majority had a progression of their melanoma, despite checkpoint inhibitor treatment (Table 1). Eight patients' melanomas were treated in an adjuvant setting after complete resection (or radiation) and could not be evaluated by the RECIST due to lack of tumor lesions.

\section{Clinical Presentation}

Clinical features of the patients according to colitis grade are given in Table 2 . Of the 33 included patients
Table 1. Tumor stage and checkpoint inhibitor treatment

\begin{tabular}{ll}
\hline & $n(\%)$ \\
\hline Total number of patients & $33(100)$ \\
Age, median (range) & $59(30-86)$ \\
Male & $22(67)$ \\
Female & $11(33)$ \\
Metastatic melanoma & \\
$\quad$ Cancer stage ${ }^{\mathrm{a}}$ III & $11(33)$ \\
$\quad$ Cancer stage ${ }^{\mathrm{a}}$ IV & $22(67)$ \\
Checkpoint inhibitor & \\
$\quad$ Ipilimumab & $19(58)$ \\
Ipilimumab + pembrolizumab & $2(6)$ \\
$\quad$ Ipilimumab + nivolumab & $8(24)$ \\
$\quad$ Pembrolizumab & $3(9)$ \\
Nivolumab & $1(3)$ \\
Tumor response & \\
$\quad$ Progressive disease & $18 / 25(72)$ \\
Partial response & $3 / 25(12)$ \\
Stable disease & $0 / 25(0)$ \\
Complete response & $4 / 25(16)$ \\
\hline
\end{tabular}

Data are presented as number (\%), unless otherwise stated. a American Joint Committee on Cancer (AJCC). Tumor stages are given in the Methods section. ${ }^{\mathrm{b}}$ Response evaluation criteria in solid tumors (RECIST).

with colitis, 25 received a complete colonoscopy and another 2 patients received a flexible sigmoidoscopy up to the left colonic flexure. Details on endoscopic findings are presented in Table 3, and representative endoscopic im- 
Table 2. Colitis grade 3 and 4

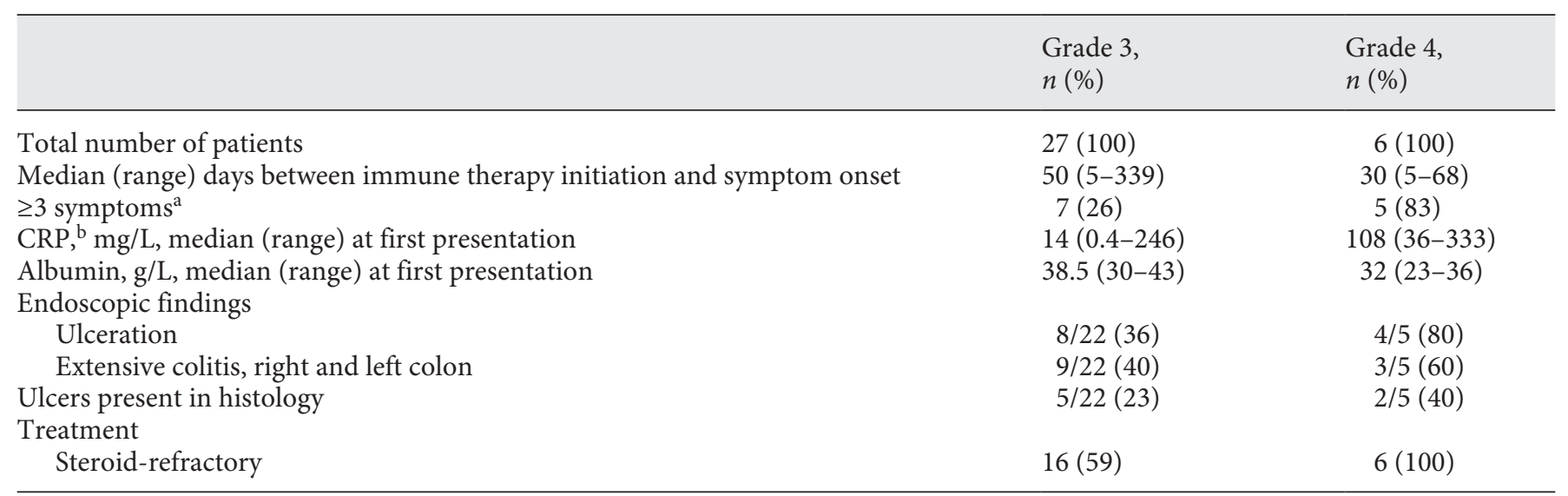

Data presented as $n(\%)$, unless otherwise stated. a Symptoms: diarrhea, bloody stool, abdominal pain, fever $\left(>38.2{ }^{\circ} \mathrm{C}\right)$, emesis, and peritoneal signs. ${ }^{\mathrm{b}} \mathrm{CRP}, \mathrm{C}$-reactive protein.

Fig. 2. Representative colonoscopy findings (classified by the endoscopist) in patients with ICIAC. a Mild erythema and small erosions. b Extensive colitis with superficial clean-based ulcers. c Extensive colitis with deep ulcerations. d Moderate erosions, with histology showing deep ulceration into the submucosa.

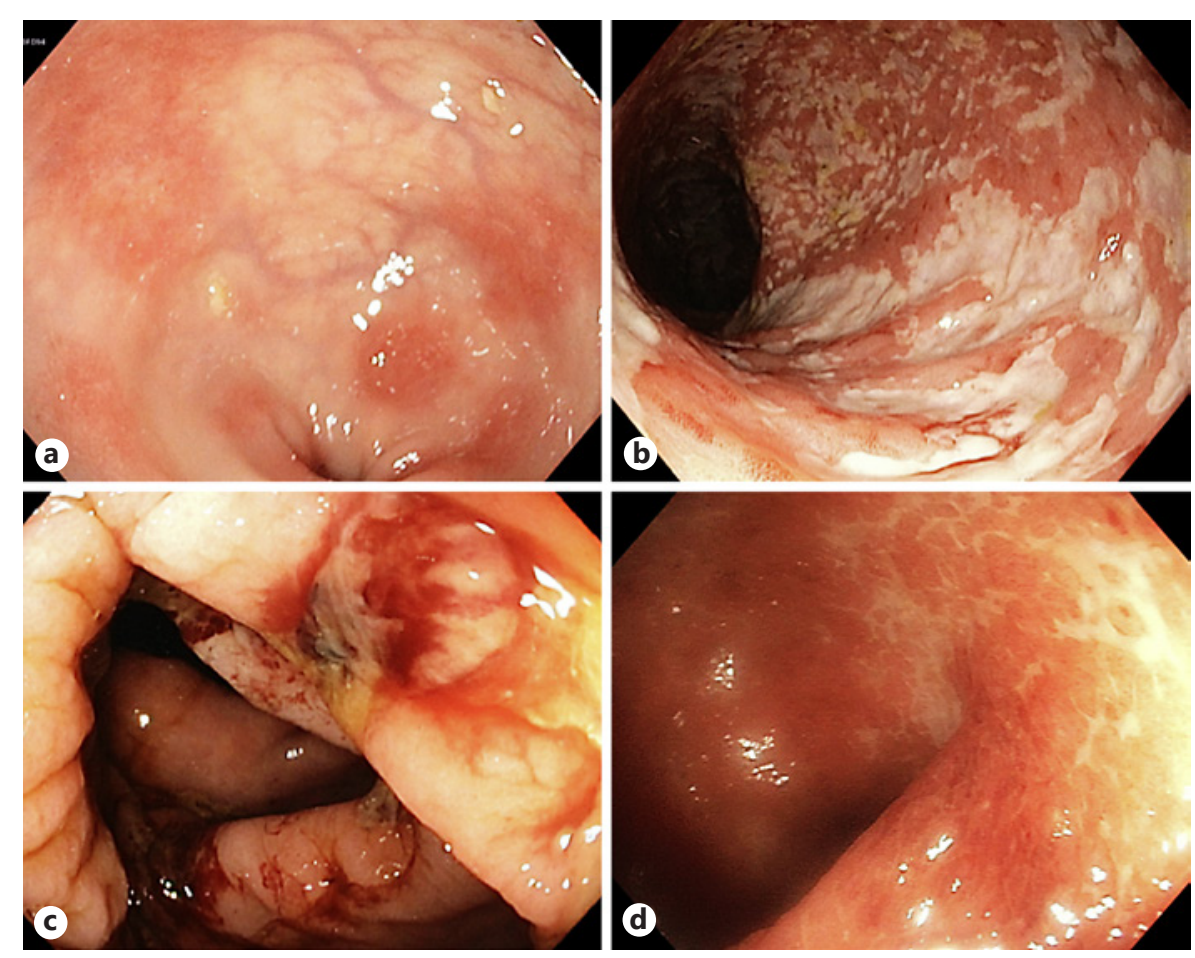

ages are shown in Figure 2. Two patients did not show visible signs of colitis: histopathological analysis of 1 patient confirmed inflammation, while in the other patient, modest lymphocytic infiltrates in conjunction with elevated fecal calprotectin was found to confirm the diagnosis of colitis. Histological features present in colitis consisted of immune cell infiltration, cryptitis, crypt abscesses, apoptosis, and ulcerations.
Patients with colitis grade 4 had significantly higher CRP and lower albumin values (Table 2). There was no difference between the endoscopic extents of inflammation. However, it appeared that grade 4 colitis patients slightly more often developed a steroid-refractory disease course (Table 3 ). 
Table 3. Clinical findings

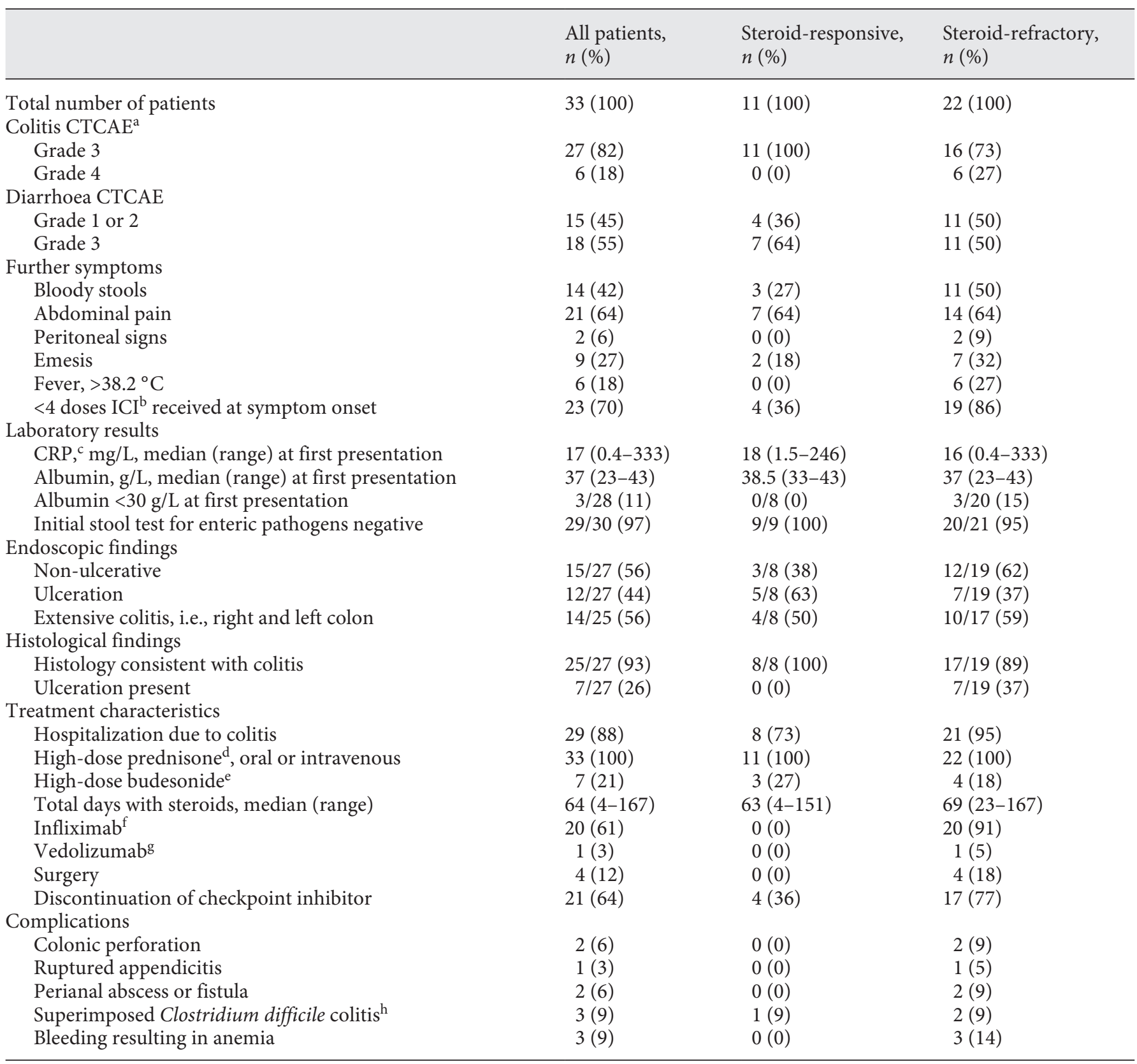

Data presented as $n$ (\%), unless otherwise stated. ${ }^{a}$ CTCAE, Common Terminology Criteria for Adverse Events. ${ }^{\mathrm{b}}$ ICI, immune checkpoint inhibitor. ${ }^{\mathrm{c}} \mathrm{CRP}, \mathrm{C}$-reactive protein. ${ }^{\mathrm{d}}$ High-dose prednisone: $>1 \mathrm{mg} / \mathrm{kg}$ body weight. ${ }^{\mathrm{e}}$ High-dose budesonide: $>9 \mathrm{mg} .{ }^{\mathrm{f}} 5 \mathrm{mg} / \mathrm{kg}$ body weight. ${ }^{\mathrm{g}} 2 \times 300 \mathrm{mg} \mathrm{IV}$. $^{\mathrm{h}}$ All successfully treated with metronidazole.

\section{Treatment of Colitis}

Detailed clinical features of the patients according to steroid-responsive versus steroid-refractory cases are given in Table 3 . The majority of patients $(88 \%)$ had to be hospitalized for treatment. All patients received ste- roids as first-line therapy. Twenty-seven patients initially received high-dose prednisone $(>1 \mathrm{mg} / \mathrm{kg}$ body weight). The remaining 6 patients initially receiving a lower prednisone dose required a dose escalation. In 21 patients, the checkpoint inhibitor was discontinued - 
significantly more often in steroid-refractory cases. The remaining 12 patients had either already completed their 4 cycles of therapy or discontinued therapy due to cancer progression or a different irAE. Other than colitis and diarrhea, common irAEs were skin rash (42\%), thyroiditis (18\%), hypophysitis, and pancreatitis (each $12 \%)$. Furthermore, less frequent irAEs such as hepatitis, pneumonia, myocarditis, and uveitis were observed (each $\leq 6 \%$ ). In 8 patients on a combination regime (6 with ipilimumab/nivolumab and 2 with ipilimumab/ pembrolizumab), only ipilimumab treatment was stopped. This was sufficient for symptom control in conjunction with the initiation of corticosteroids. Seven patients (21\%) received high-dose budesonide ( $>9 \mathrm{mg}$, oral or rectal foam), of which 3 received budesonide in order to taper prednisone. The median duration of steroid treatment was 64 days (Table 3). In the absence of symptom control ("steroid-refractory" cases), immunosuppressive treatment was intensified after a median of 13 days. Fourteen patients received a single infusion of infliximab (6 patients received up to 3 infusions). Vedolizumab was used to treat steroid-refractory colitis in 1 patient, who additionally suffered from multiple myeloma. The decision was made after consultation with hematologists, who advised against the use of infliximab. In addition, prednisone was tapered as slow as over 158 days in this patient.

\section{Features of Steroid-Responsive versus Steroid-}

Refractory Colitis

Most clinical, laboratory, and endoscopic findings did not differ between steroid-responsive and steroid-refractory patients (Table 3 ). In steroid-refractory cases, it appeared that colitis more often occurred before completing 4 cycles of checkpoint inhibitor therapy (Table 3 ). In addition, fever was more often reported, while abdominal pain, bloody stools and emesis were not different compared to steroid-responsive cases (Table 3). Of note, none of the steroid-responsive patients had colitis grade 4 , while $6(27 \%)$ of steroid-refractory patients had grade 4 colitis (5 received infliximab, 3 were colectomized, ref. Fig. 1). Of the steroid-refractory patients, 5 developed complications: 2 suffered from severe gastrointestinal hemorrhage requiring transfusion, one developed a fistula, and 2 underwent surgery due to sigmoid perforation. A very low albumin of $<30 \mathrm{~g} / \mathrm{L}$ was only seen in steroid-refractory patients (Table 3 ).

Histologically detected ulcerations, which infiltrated deep into the submucosa, were only present in steroidrefractory patients and were also detected in the afore- mentioned colectomy specimens. Six of the 7 patients with histologically diagnosed ulcerations also had macroscopic findings described as ulcers by the endoscopist. In 1 case, moderate erosions without macroscopic ulcerative inflammation were described at endoscopy (ref. Fig. 2d). However, histological analysis of biopsies revealed an ulcerative colitis with lesions extending beyond the muscularis mucosae, thus being in agreement with the definition of histologic ulcerations.

\section{Course of Melanoma Treatment}

Regarding oncological response, the frequency of patients responding to the checkpoint inhibitor therapy (partial or complete) was higher in the steroid-refractory group (27 vs. $9 \%)$. The frequency of patients with tumor progression did not differ between steroid-responsive and -refractory cases (55\% in both groups). Eight patients were treated with an anti-PD1 antibody (pembrolizumab) following the treatment with ipilimumab. Of those, 1 patient developed recurrent intestinal irAE (diarrhea grade III and colitis grade III). Another patient previously treated with ipilimumab received additional cycles of ipilimumab plus nivolumab and likewise developed recurrent colitis (diarrhea grade III and colitis grade II).

\section{Discussion}

In the present study, we analyzed clinical features, management, and outcomes of 33 melanoma patients suffering from severe checkpoint inhibitor-induced colitis. Patients were treated with steroids in the first-line, followed by infliximab or vedolizumab in the secondline. Consistent with more severe inflammation, patients with a CTCAE grade 4 colitis had higher CRP and lower albumin levels. Steroid-refractory cases had to discontinue checkpoint inhibitors more often. In addition, histologically diagnosed ulcerations were only present in steroid-refractory patients.

All patients in our study presented with diarrhea. Additional symptoms, such as bloody stools, emesis, fever, albumin $<30 \mathrm{~g} / \mathrm{L}$, higher CTCAE colitis grade, and complications, appeared to be more often in patients with a steroid-refractory course. In contrast to earlier studies, we did not detect endoscopic features connected to a steroid-refractory disease course $[16,17]$. Especially, macroscopically visible ulcerations were proposed to predict a steroid-refractory course $[16,18]$. However, our findings did not confirm this. Of all analyzed patients, $63 \%$ of steroid-responsive patients had ulcerations, while only $37 \%$ 
of steroid-refractory patients had a colitis with macroscopically visible ulcers.

Of note, we detected an important histological difference between steroid-refractory and -responsive patients. Deep histologically present ulcerations invading the submucosa were only present in steroid-refractory cases. This was also detected in severe cases, leading to colectomy. To the best of our knowledge, the predominance of such ulcerations in steroid-refractory cases has not been reported in earlier studies investigating checkpoint inhibitor-induced colitis. A recent study suggested a rapid colonoscopy ( $<7$ days after colitis onset) because this decreases the overall duration of symptoms, steroid treatment, and hospitalization rates [19]. In this context, our finding would further support early endoscopy in patients with grade 3 or 4 checkpoint inhibitor-induced colitis. Especially, if histological ulcerations are detected early, this could reduce the disease burden and complication rate by early steroid cessation and application of infliximab or vedolizumab. In our study, we confirmed the prompt effect and good tolerability of infliximab for the treatment of checkpoint inhibitor-induced colitis. Twenty patients were treated with infliximab, and 14 patients needed only one infliximab cycle $(5 \mathrm{mg} / \mathrm{kg})$. None of the patients developed side effects, and we did not observe an infliximab-resistant case. Infliximab resistance may be caused by cytomegalovirus (CMV) infection [20]. Seventeen of the 33 patients were evaluated for CMV by immunohistochemistry, and all were negative (data not shown).

Recent literature shows no evidence indicating that the immunosuppressive treatment for colitis does attenuate the anti-neoplastic efficacy of ICI treatment [21, 22]. Most patients need only one dose of infliximab, and early administration could facilitate fast steroid tapering. During our follow-up (i.e., until complete remission of ICIAC), we did not see a worse tumor response in our steroid-refractory cohort. However, the influence of antiTNFa biologics on the efficacy of ICI therapy has to be evaluated in larger prospective trials with longer followup comparing survival outcomes.

The presence of irAEs with checkpoint inhibitor therapy has been identified as a positive prognostic factor for the probability of tumor response, because irAEs might serve as a surrogate for immune system activation by checkpoint inhibitor therapy $[23,24]$. In line with this, we observed a better tumor response (partial or complete) in the steroid-refractory group (27 vs. $9 \%$ ). In the steroidresponsive group, we detected a resolution of colitis with cessation of ipilimumab. These 8 patients instead received pembrolizumab. This is in line with the literature, showing a higher incidence of colitis with anti-CTLA-4 therapy than anti-PD1 antibodies $[10,11,25]$.

Our study included only patients who were treated in the Department of Gastroenterology and Hepatology. Our results may therefore be biased toward more severe cases, as mild cases of irAEs are usually treated by dermatologists themselves. This might explain the reported severity of symptoms, high CTCAE grades, and the prevalence of complications, as a comparable recent study detected a need for surgery in $3 / 60$ patients (vs. $2 / 33$ patients in our study) [26]. Furthermore, it also explains why such a high number of 22 patients (67\%) presented with a steroid-refractory disease course needed additional treatment with infliximab or even surgery. And it might also explain why the majority of patients had cancer progression despite severe colitis, which is in contrast to an earlier study investigating the disease course in patients with checkpoint inhibitor-induced colitis [26].

Checkpoint inhibitor therapy has evolved as a mainstay in the treatment of multiple cancer indications within the past few years. With its growing application, the incidence of irAEs such as diarrhea and colitis has increased as well, and gastroenterologists are more likely to encounter such toxicities. We demonstrated that ICIAC can lead to severe complications, more often seen in steroid-refractory patients. Therefore, factors predictive for a steroid-refractory disease course are important to be identified. As histologically detected deep ulcerations were only present in steroid-refractory patients, such ulcerations could aid identifying patients for a failure of steroid-treatment. Prompt endoscopy leading to early infliximab initiation and corticosteroid cessation might shorten symptoms and accelerate colitis resolution. However, this needs to be validated in larger and/or prospective studies.

\section{Statement of Ethics}

This study was approved by the Cantonal ethics committee of the Canton Zurich (Project-ID 2018-00860) and is written according to the principles of good clinical practice.

\section{Disclosure Statement}

The authors have no conflicts of interest related to this study to declare. 


\section{Funding Sources}

This work was supported by research grants from the Stiftung Experimentelle Biomedizin to M.S., the Monique Dornonville de la Cour Stiftung to M.S. and the Swiss National Science Foundation to M.S. [Grant Nos. 314730_166381 and 320030_184753].

\section{Author Contributions}

J.B. and S.B.: data analysis and drafting of the manuscript. L.B., R.D., M.P.L., M.J.B., S.B., C.G., B.M., G.R., and M.S.: patient recruitment and care, and revision of the manuscript. M.S.: study design and supervision. All authors approved the submitted version of the manuscript.

\section{References}

1 Pardoll DM. The blockade of immune checkpoints in cancer immunotherapy. Nat Rev Cancer. 2012;12(4):252-64.

2 Pico de Coaña Y, Choudhury A, and Kiessling R. Checkpoint blockade for cancer therapy: revitalizing a suppressed immune system. Trends Mol Med. 2015;21(8):482-91.

3 Sunshine J and Taube JM. PD-1/PD-L1 inhibitors. Curr Opin Pharmacol. 2015;23:32-8.

4 Hodi FS, O'Day SI, McDermott DF, Weber RW, Sosman JA, Haanen JB, et al. Improved survival with ipilimumab in patients with metastatic melanoma. N Engl J Med. 2010 363(8):711-23.

5 Robert C, Thomas L, Bondarenko I, O’Day S, Weber J, Garbe C, et al. Ipilimumab plus dacarbazine for previously untreated metastatic melanoma. N Engl J Med. 2011;364(26): 2517-26.

6 Motzer RJ, Escudier B, McDermott DF, George S, Hammers HJ, Srinivas S, et al. Nivolumab versus everolimus in advanced renal-cell carcinoma. N Engl J Med. 2015; 373(19):1803-13

7 Reck M, Rodríguez-Abreu D, Robinson AG Hui R, Csőszi T, Fülöp A, et al. Pembrolizumab versus chemotherapy for PD-L1-positive non-small-cell lung cancer. N Engl J Med. 2016;375(19):1823-3.

8 Bellmunt J, de Wit R, Vaughn DJ, Fradet Y, Lee JL, Fong L, et al. Pembrolizumab as second-line therapy for advanced urothelial carcinoma. N Engl J Med. 2017;376(11):101526.

9 Rittmeyer A, Barlesi F, Waterkamp D, Park K, Ciardiello F, von Pawel J, et al. Atezolizumab versus docetaxel in patients with previously treated non-small-cell lung cancer (OAK): a phase 3, open-label, multicentre randomised controlled trial. Lancet. 2017;389(10066): 255-65.

10 Ibrahim RA, Berman DM, DePril V, Humphrey RW, Chen T, Messina M, et al. Ipilimumab safety profile: summary of findings from completed trials in advanced melanoma. J Clin Oncol. 2011;29:8583.
11 Larkin J, Chiarion-Sileni V, Gonzalez R, Grob JJ, Cowey CL, Lao CD, et al. Combined nivolumab and ipilimumab or monotherapy in untreated melanoma. N Engl J Med. 2015; 373(1):23-34.

12 Boutros C, Tarhini A, Routier E, Lambotte O, Ladurie FL, Carbonnel F, et al. Safety profiles of anti-CTLA-4 and anti-PD-1 antibodies alone and in combination. Nat Rev Clin Oncol. 2016;13(8):473-86.

13 De Felice KM, Gupta A, Rakshit S, Khanna S, Kottschade LA, Finnes HD, et al. Ipilimumab-induced colitis in patients with metastatic melanoma. Melanoma Res. 2015;25(4): 321-7.

14 Marsden JR, Newton-Bishop JA, Burrows L, Cook M, Corrie PG, Cox NH, et al. Revised U.K. guidelines for the management of cutaneous melanoma 2010. Br J Dermatol. 2010; 163(2):238-56.

15 Haanen J, Carbonnel F, Robert C, Kerr K, Peters S, Larkin J, et al., ESMO Guidelines Committee. Management of toxicities from immunotherapy: ESMO Clinical Practice Guidelines for diagnosis, treatment and follow-up. Ann Oncol. 2017;28(suppl_4):iv119-iv142. 10.1093/annonc/mdx225. 28881921.

16 Geukes Foppen MH, Rozeman EA, van Wilpe S, Postma C, Snaebjornsson P, van Thienen $\mathrm{JV}$, et al. Immune checkpoint inhibition-related colitis: symptoms, endoscopic features, histology and response to management. ESMO Open. 2018;3(1):e000278.

17 Wang Y, Abu-Sbeih H, Mao E, Ali N, Qiao W, Trinh VA, et al. Endoscopic and histologic features of immune checkpoint inhibitor-related colitis. Inflamm Bowel Dis. 2018;24(8): 1695-705.

18 Jain A, Lipson EJ, Sharfman WH, Brant SR, and Lazarev MG. Colonic ulcerations may predict steroid-refractory course in patients with ipilimumab-mediated enterocolitis. World J Gastroenterol. 2017;23(11):2023-8.
19 Abu-Sbeih H, Ali FS, Luo W, Qiao W, Raju GS, and Wang Y. Importance of endoscopic and histological evaluation in the management of immune checkpoint inhibitor-induced colitis. J Immunother Cancer. 2018; 6(1):95.

20 Franklin C, Rooms I, Fiedler M, Reis $\mathrm{H}$ Milsch L, Herz S, et al. Cytomegalovirus reactivation in patients with refractory checkpoint inhibitor-induced colitis. Eur J Cancer. 2017;86:248-56.

21 Badran YR, Cohen JV, Brastianos PK, Parikh AR, Hong TS, Dougan M. Concurrent therapy with immune checkpoint inhibitors and TNFa blockade in patients with gastrointestinal immune-related adverse events. J Immunother Cancer. 2019;7(1):226.

22 Schadendorf D, Wolchok JD, Hodi FS, Chiarion-Sileni V, Gonzalez R, Rutkowski P, et al. Efficacy and safety outcomes in patients with advanced melanoma who discontinued treatment with nivolumab and ipilimumab because of adverse events: a pooled analysis of randomized phase II and III trials. J Clin Oncol. 2017;35:3807-14.

23 Downey SG, Klapper JA, Smith FO, Yang JC, Sherry RM, Royal RE, et al. Prognostic factors related to clinical response in patients with metastatic melanoma treated by CTL-associated antigen- 4 blockade. Clin Cancer Res. 2007;13(22 Pt 1):6681-8.

24 Fujii T, Colen RR, Bilen MA, Hess KR, Hajjar $\mathrm{J}$, Suarez-Almazor ME, et al. Incidence of immune-related adverse events and its association with treatment outcomes: the MD Anderson Cancer Center experience. Invest New Drugs. 2018;36:638-46.

25 Robert C, Schachter J, Long GV, Arance A, Grob JJ, Mortier L, et al. Pembrolizumab versus ipilimumab in advanced melanoma. N Engl J Med. 2015;372(26):2521-32.

26 Hughes MS, Zheng H, Zubiri L, Molina GE, Chen ST, Mooradian MJ, et al. Colitis after checkdpoint blockade: a retrospective cohort study of melanoma patients requiring admission for symptom control. Cancer Med. 2019; 8(11):4986-4999. 